\title{
Colloquium
}

\section{Unified scaling law for earthquakes}

\author{
Kim Christensen*t, Leon Danon*, Tim Scanlon*, and Per Bak ${ }^{\dagger \neq}$ \\ *Blackett Laboratory, Imperial College, Prince Consort Road, London SW7 2BW, United Kingdom; and ₹Department of Mathematics, Imperial College, \\ Queen's Gate, London SW7 2BZ, United Kingdom
}

We propose and verify a unified scaling law that provides a framework for viewing the probability of the occurrence of earthquakes in a given region and for a given cutoff magnitude. The law shows that earthquakes occur in hierarchical correlated clusters, which overlap with other spatially separated correlated clusters for large enough time periods and areas. For a small enough region and time-scale, only a single correlated group can be sampled. The law links together the Gutenberg-Richter Law, the Omori Law of aftershocks, and the fractal dimensions of the faults. The Omori Law is shown to be the short time limit of general hierarchical phenomenon containing the statistics of both "main shocks" and "aftershocks," indicating that they are created by the same mechanism.

E arthquakes are a complicated spatiotemporal phenomenon which is poorly understood by looking at individual isolated events. It is important to study large space and time correlations of many events. To this end, it has been suggested that earthquakes are a critical phenomenon (1). The most striking evidence for this suggestion is the observation of the distribution of earthquake magnitudes, known as the Gutenberg-Richter Law (2). There is also an observed power-law distribution of "aftershocks," the Omori Law (3). In particular we note the following:

- The distribution of the size of earthquakes follows the Gutenberg-Richter law (2) that states that the number of earthquakes with magnitude $\mathrm{M}$ greater than $\mathrm{m}$ is given by

$$
\log _{10} N(M>m) \propto-b m,
$$

where $b \approx 1$. Introducing a variable $S=10^{m \S}$

$$
N(S>s) \propto s^{-b} .
$$

- The short time temporal correlation between earthquakes is given by the Omori Law (3), which states that immediately after an earthquake, the frequency of a sequence of aftershocks decays with time $T$ as

$$
N(T) \propto T^{-\alpha},
$$

where $\propto \approx 1$.

- The fault systems and the spatial distribution of epicenters of earthquakes are fractal (4).

Thus the phenomena of earthquakes display a complex spatiotemporal behavior in addition to the self-similarity in the statistics of earthquakes. The temporal complexity is obviously of dynamical origin, but the statistics as well as the geometrical fractal structure displayed by the faults and by the spatial distribution of epicenters also should be explained as a result of a dynamical process. One might speculate whether it is possible to unify these observations.

Aftershocks are described as correlated events that occur after a large seismic event. This definition is widely used to separate catalogs of earthquake data into aftershocks and "main shocks." The Omori Law (3) is based on classifying earthquakes in this manner, and it has led to the commonly held belief that aftershocks are created by a mechanism different from the cause of main shocks. Gardner and Knopoff (5) suggested, quite reasonably, that an earthquake should be characterized as an aftershock if it belongs to a cluster of correlated events and as a main shock if it belongs to a series of "isolated" random events with Poisson statistics. However, as we shall demonstrate, there seems to be no rigorous scientific method to distinguish whether an earthquake is an aftershock or a main shock, because, according to this definition, the categorization depends on the length scale, magnitude, and time scale considered. More specifically, we show that the temporal intervals between earthquakes in California obey a scaling law that spans over eight orders of magnitude, expressing in a compact way the hierarchical organization of earthquakes in time and space. There is no unique, operational way of distinguishing between main shocks and aftershocks. The proposed law unifies the GutenbergRichter Law, the Omori Law, and the law describing the fractality of fault systems. The Omori Law, which relates to the statistics of aftershocks occurring within minutes of an earthquake, is just the short time limit of a general hierarchical scaling phenomena occurring at all accessible time scales.

Because only critical phenomena exhibit scaling laws, this result supports the hypothesis that earthquakes are selforganized critical (SOC) phenomena (6-11).

\section{Analysis}

Southern California was chosen for analysis, as it is a region of high seismic activity and has a comprehensive network of earthquake detectors. Therefore, excellent earthquake catalogs with large numbers of events are available for this region.

Catalogs of Southern California earthquake data from 19842000 were used in this analysis (12). Data are available for pre-1984 earthquakes, but these were discarded as the catalogs were found to have a high proportion of unusable events for which either magnitude or location entries were missing, which would certainly bias the analysis. The remaining data covered a region from $20^{\circ} \mathrm{N}$ to $45^{\circ} \mathrm{N}$ latitude and $100^{\circ} \mathrm{W}$ to $125^{\circ} \mathrm{W}$ longitude, and contained 293,405 earthquakes.

A two-point spatial and temporal analysis was performed on this region. This analysis was carried out by splitting the region under consideration into cells of size $L \times L$, and in each of these regions, by calculating the first-return (interoccurrence) time probability defined as follows:

The first-return time probability $P_{\mathrm{S}, L}(T)$ is the probability that, if an earthquake occurs in a given region at time $T_{0}$, another earthquake will occur at a time $T_{0}+T$.

This paper results from the Arthur M. Sackler Colloquium of the National Academy of Sciences, "Self-Organized Complexity in the Physical, Biological, and Social Sciences," held March 23-24, 2001, at the Arnold and Mabel Beckman Center of the National Academies of Science and Engineering in Irvine, CA.

${ }^{\dagger}$ To whom reprint requests should be addressed. E-mail: k.christensen@ic.ac.uk or bak@alf.nbi.dk.

$\S S$ may be related to seismic moment $S^{\prime}$ by $S=S^{\prime 2 / 3}$. 

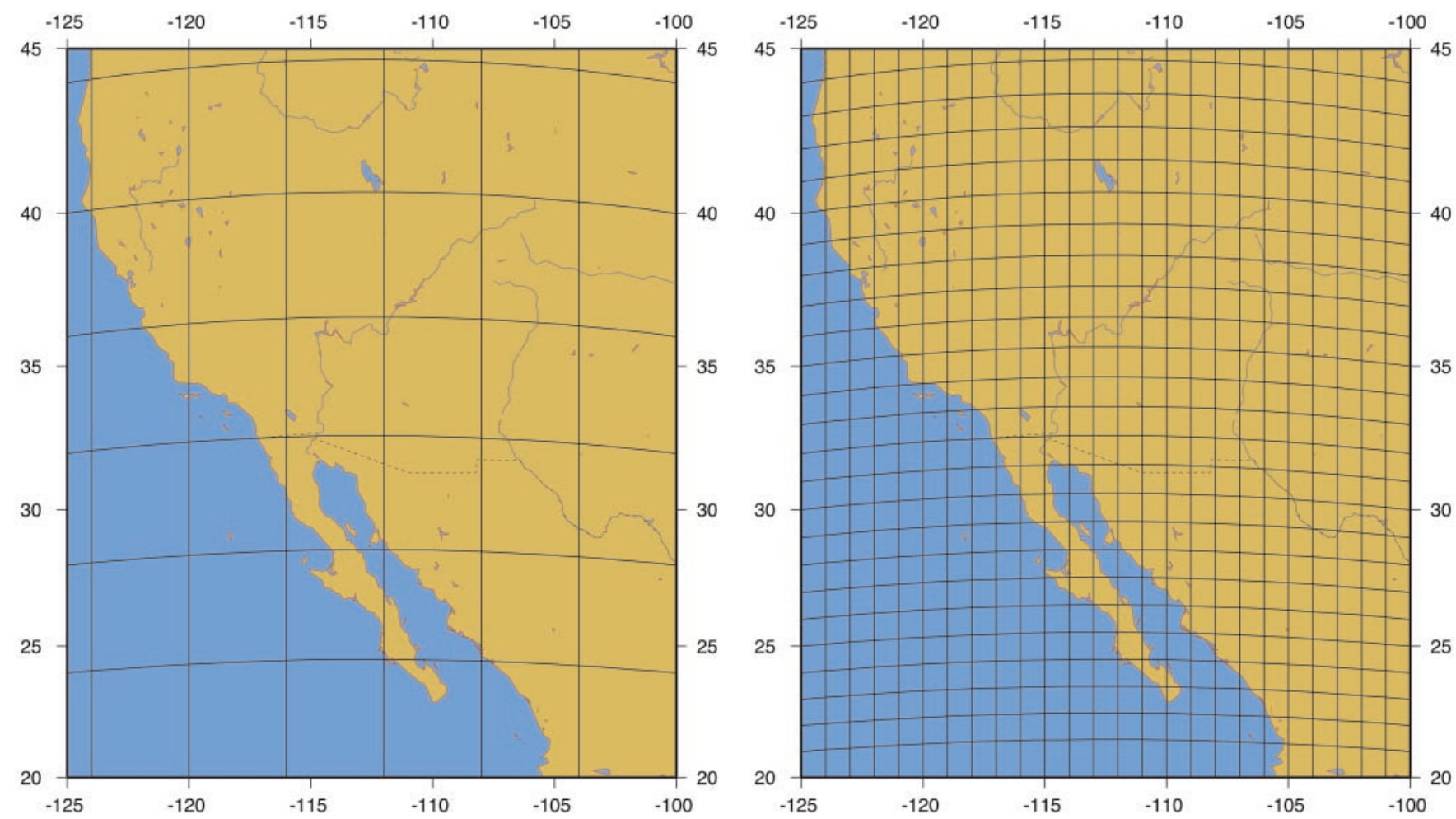

Fig. 1. Southern California seismic region covered by a grid of cells of $4^{\circ} \times 4^{\circ}$ (Left) and cells of $1^{\circ} \times 1^{\circ}\left(\approx 111 \times 111 \mathrm{~km}^{2}\right)($ Right $)$.

The analysis was performed for varying cell sizes $L$, and varying cutoff magnitudes $S$, where only earthquakes with magnitude greater than the cutoff magnitude $S$ were used.

The analysis required the latitude, longitude, magnitude, and occurrence time of each earthquake, with the earthquakes ordered chronologically. This analysis was carried out by a program that read in the data from the 17 catalogs spanning the years 1984-2000. The program located the time, latitude, and longitude of each earthquake, then converted them into decimal values, taking care to account for leap years and defining 00:00 h on January 1, 1984 as $t=0$. The earthquakes were sorted to ensure that they were in chronological order, and then the time, local magnitude, latitude, and longitude were output to a file. There were 24,469 events that had a null value for one or more of their attributes, and as such were discarded at this stage.

To calculate the magnitude distribution of earthquakes, the earthquake data were read through for incrementally increasing values of magnitude $m$, and the number of earthquakes with magnitude $M$ greater than the cutoff magnitude $m$ were counted. The resulting distribution of the number of earthquakes $N$ with $(M>m)$ divided by the number of years, is then a distribution of the number of earthquakes with a magnitude greater than $m$ per year.

The analysis for the first-return time correlation function required the area of Southern California to be divided up into equally sized regions of area $L \times L$. Fig. 1 shows the region of Southern California divided into grids of two different cell sizes.

An array was then defined whose individual elements correspond to a specific cell in the Southern California region and contain the time of the previous earthquake in that cell. For varying cell sizes and cutoff magnitudes, all of the earthquakes were read through in chronological order, following these simple rules.

- The earthquake's data were read in and used if its magnitude was greater than the cutoff magnitude.
- The earthquake's coordinates were used to place it within a particular grid cell.

- The time of the last earthquake in that region was read from the array (if it was the first earthquake to have occurred in that region, the earthquake's time was stored in the empty array space, and the next earthquake was read in).

- The time between the two earthquakes was calculated and put into the appropriate bin.

- The array was updated to the new earthquake's time.

The analysis was repeated for the cell sizes $L=0.25^{\circ}, 0.5^{\circ}, 1^{\circ}$, $2^{\circ}, 4^{\circ}$, and for the cutoff magnitudes 2,3 , and 4 . The degrees of cell size is related to distance as $1^{\circ} \approx 111 \mathrm{Km}$. Using smaller cell sizes and higher magnitude cutoffs would lead to a small number of events in each cell, thus giving poor statistics.

The results were best observed in the form of a probability distribution with the interoccurrence times placed into appropriate bins. However, the values ranged from a few milliseconds to a few million seconds ( 17 years). Obviously, this range is a very large one, so it would have been extremely impractical to put these values in equal-sized bins; instead, bins with exponentially increasing sizes were used. A value of 2.5 was used for the exponential base of the bin boundaries, which gave 27 bins for this range.

\section{Results}

Fig. 2 shows the magnitude distribution for the Southern California region, which is the number of earthquakes per year with magnitude $(M>m)$. The dashed red line shows the GutenbergRichter Law with a gradient $b \approx 1$. There is a deficit for $m \leq 2$ due to problems associated with detecting small earthquakes. Therefore, only earthquakes with $m \geq 2$ are considered in the following analysis.

Fig. 3 shows $P_{\mathrm{S}, \mathrm{L}}(T)$, the distribution of the interoccurrence times between consecutive earthquakes for a given cutoff magnitude $m$ [where $\left.m=\log _{10}(S)\right]$ in a region size $L \times L$. 


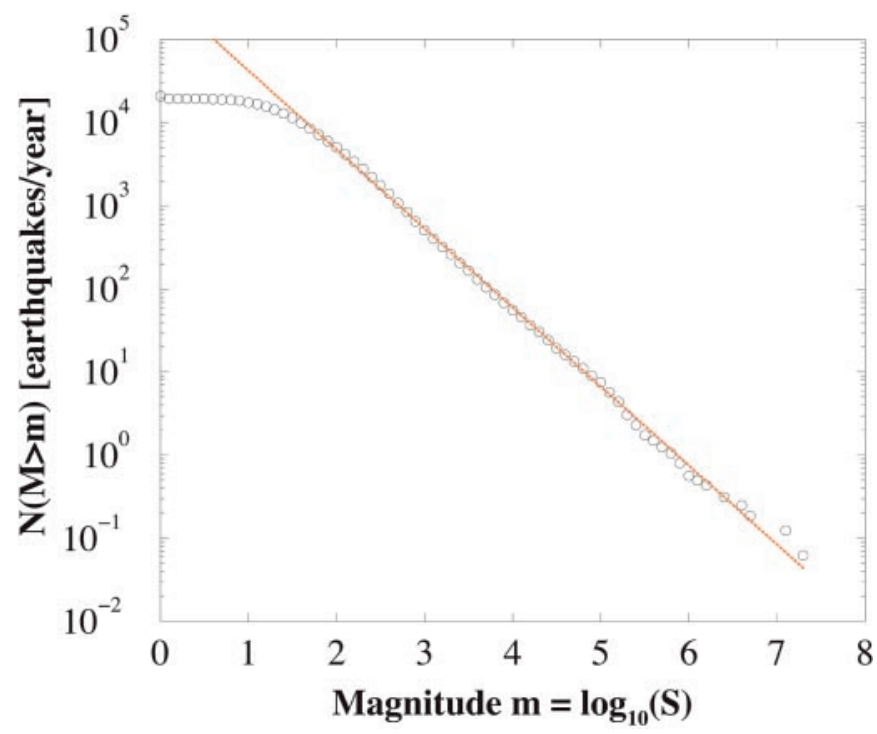

Fig. 2. Earthquake magnitude distribution showing a power-law behavior over 6 decades. The graph follows $\log _{10} N(M>m) \propto-b m$, where $b$ is the Gutenberg-Richter exponent $b=1$ (dashed red line). The roll-off for $m<2$ is due to difficulties with detecting very small earthquakes.

For interoccurrence times below $40 \mathrm{~s}$, earthquakes overlap, and there is difficulty in resolving separate events. This fact accounts for the deficit for small $T$; so, in the following, only the interoccurrence times for $T>38 \mathrm{~s}$ will be considered (38 s corresponds to the crossover between two bins).

The graphs all show a power law regime that corresponds to the Omori Law of the decay of the frequency of aftershocks $\propto T^{-\alpha}$, where $\alpha \approx 1$. It is important to note that the range of the power-law region varies with cutoff magnitude and cell size.

Examining the graph closely shows that for fixed region size $L$ but increasing cutoff $S$, the range of the power-law regime

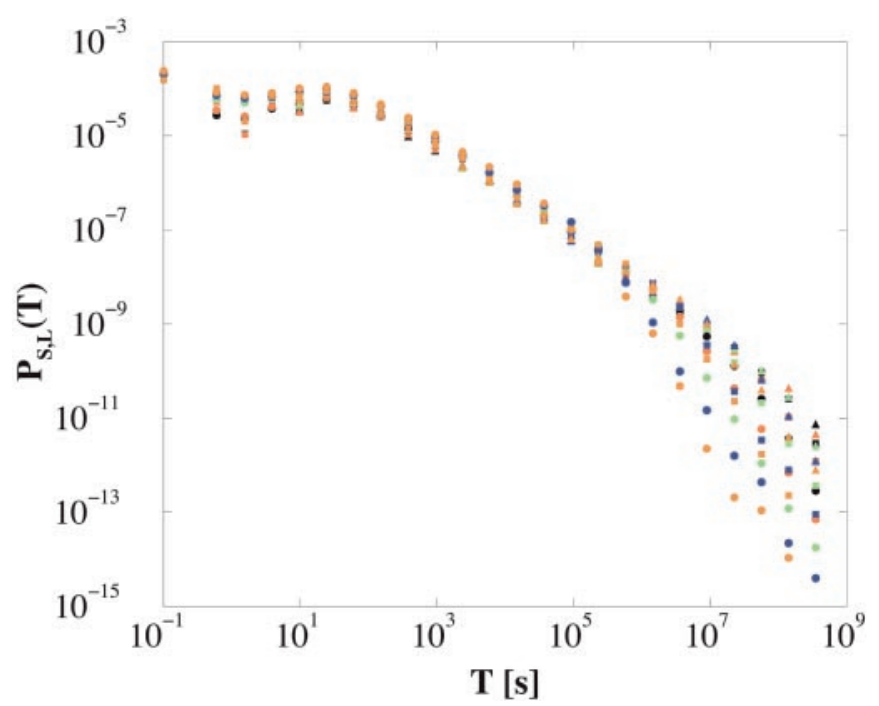

Fig. 3. The distribution $P_{S, L}(T)$ of interoccurrence times $T$ with magnitude greater than $S$. The solid circles, squares, and triangles correspond to cutoffs $m=2,3$, and 4 , respectively. The color coding represents the linear size $L=$ $0.25^{\circ}$ (black), $0.5^{\circ}$ (red), $1^{\circ}$ (green), $2^{\circ}$ (blue), and $4^{\circ}$ (orange) of the cells covering Southern California. For $T<\mathbf{4 0} \mathrm{s}$, earthquakes overlap, and individual earthquakes cannot be resolved. This result causes the deficit for small $T$, so only intervals $T>38 \mathrm{~s}$ will be considered.

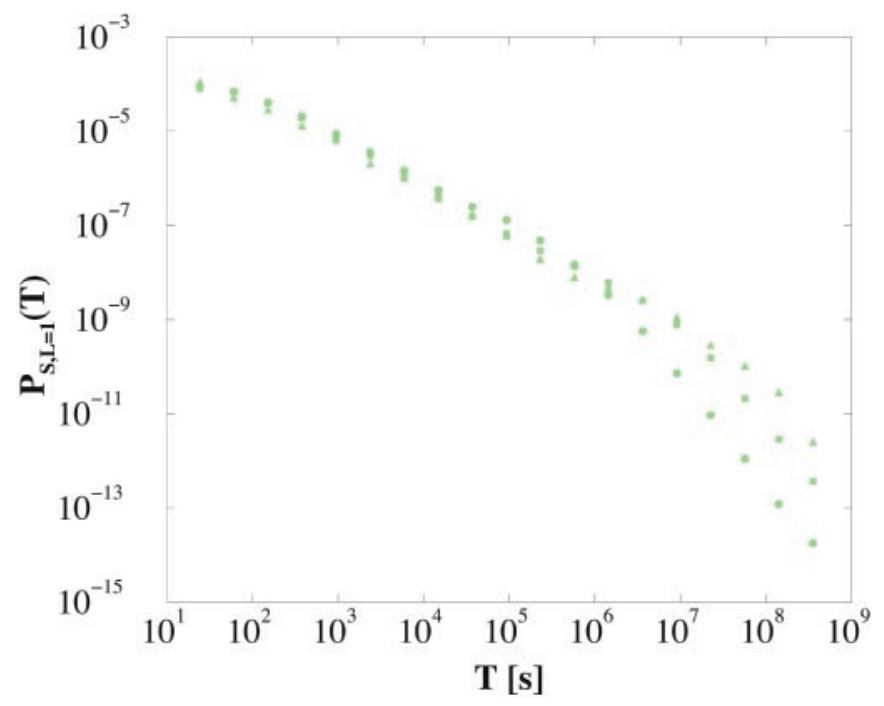

Fig. 4. First-return (interoccurrence) time distributions for $L=1^{\circ}$ and $m=2$, 3, and 4. $P_{S, L}=1^{\circ}(T)$ follows a power law $P_{S, L=1}{ }^{\circ}(T) \propto T^{-\alpha}$ where $\alpha \approx 1$. The Omori Law region increases with increasing $m$.

increases, as shown in Fig. 4. In other words, the power-law cutoff scales with $S$ and can be expressed as

$$
P_{S, L}=1^{\circ}(T) \propto T^{-\alpha} g\left(T S^{-b}\right)
$$

where $b$ is a critical exponent characterizing the distribution of earthquakes and $g(x)$ is a scaling function. Such functions are well known in the theory of critical phenomena (13), and they typically consist of a constant part for small arguments and a rapidly decaying (normally exponential) part for large arguments.

Similar behavior is seen when varying $L$ (see Fig. 5). As $L$ is increased, the length of the power-law region is reduced, which can be expressed as

$$
P_{S=100, L}(T) \propto T^{-\alpha} g^{\prime}\left(T L^{d_{f}}\right)
$$

where $g^{\prime}$ is a scaling function and $d_{f}$ is a spatial dimension exponent.

\section{The Unified Earthquake Law}

By physical arguments discussed later, we suggest that the two scaling relations can be unified and reduced further. Eq. 6 gives the distribution of temporal intervals $T$ between earthquakes in an area of size $L \times L$, exceeding magnitude $m=\log _{10} S$.

$$
P_{S, L}(T) \propto T^{-\alpha} f\left(T L^{d_{f}} S^{-b}\right)
$$

where $\alpha, b$, and $d_{f}$ are values to be fitted and $f(x)$ is a scaling function. It is seen to remain constant for values $x<1$ and to decay rapidly for $x>1$.

To verify this Law, the curves in Fig. 3 are replotted in terms of rescaled coordinates where the $x$ axis is chosen as $x=T S^{-b} L^{d f}$, and the $y$ axis represents $y=T^{\alpha} P_{S, L}(T)$. The resulting graph shown in Fig. 6 clearly shows that all of the data collapse neatly onto a single curve. Experimentally fitting the critical indices $\alpha$, $b$, and $d_{f}$ to achieve the best data collapse yielded the values $\alpha \approx$ $1, b \approx 1$, and $d_{f} \approx 1.2$. Astonishingly enough, these values, which are independently fitted for the best data collapse, directly relate to the key values describing earthquake characteristics.

- $\alpha \approx 1$, relating to the interoccurrence time, is the $\alpha$ value from the Omori Law for aftershocks. 
- $b \approx 1$, relating to the magnitude distribution, is the $b$ value from the Gutenberg-Richter Law (see Fig. 2).

- $d_{f} \approx 1.2$, relating to the cell size, may be interpreted as the effective fractal dimension of the San Andreas fault system (4).

The data collapse verifies the Law. It consists of a straight, constant part, followed by a decaying part separated by a sharp kink. The constant part relates to a highly correlated region of power-law behavior seen in Fig. 3, which corresponds to the Omori Law. This region is constant as $P_{S, L}(T)$ has been multiplied by the interoccurrence times $T^{\alpha}$. Any deviation from the power-law regime would show up dramatically in this type of plot, but it is seen to be constant over approximately eight orders of magnitude. The decaying part of the graph for large $x$ is consistent with an exponential decay, indicating a random Poisson distribution of interoccurrence times.

The highly correlated constant region on the left hand side of the kink in Fig. 6 is representative of the Omori Law in which a sequence of earthquakes are temporally correlated. The rapidly decaying part is indicative of earthquakes that would be classified as uncorrelated events and would be seen as responsible for creating the proceeding sequence of correlated earthquakes. However, what should be noted is that the kink that separates the uncorrelated events form the correlated events is entirely dependent on the value of $x$.

$$
x=T L^{d_{f}} S^{-b} .
$$

It is impossible to define either the uncorrelated events or the correlated events without defining a region size $(L)$, a magnitude size $\left[m=\log _{10}(S)\right]$, and a time scale $(T)$. Depending on the length scale and the magnitude cutoff, the Omori regime can range from a few seconds to tens of years. The process of dividing earthquakes into separate categories is, therefore, not unique and depends entirely on the choices of $S$ and $L$. For a given set of $(S, L)$ an earthquake may belong to a correlated cluster of events $(x<1)$, whereas for a different set $\left(S^{\prime}, L^{\prime}\right)$, the same earthquake belongs to a series of uncorrelated events $\left(x^{\prime}>1\right)$.

The correlations given by the Omori Law are just the short time limit of a general hierarchical scaling phenomenon occurring at all accessible time scales. Astonishingly, the statistics of earthquakes happening within minutes of an initial earthquake can be related to the statistics of earthquakes separated by tens of years. Typically, scientists have used time scales spanning a human lifetime, and thus the separation of seismic events into main shocks and aftershocks seems to represent an anthropocentric view of the phenomenon.

The data collapse supports the view that an earthquake is an SOC phenomenon, because only critical processes exhibit data collapse (13), known as scaling in critical phenomena. A plausible consequence of this view is that the process that creates main shocks also produces aftershocks, and so there is only one relaxation mechanism responsible for all earthquakes.

To understand the Unified Law for Earthquakes, it is essential to see what the value of $x$ represents. The quantity $L^{d f} S^{-b}$ in the scaling function represents the average number of earthquakes per unit time, with seismic moment greater than $S$ occurring in the area size $L \times L$. Therefore, $x$ is a measure of the number of earthquakes happening within a time interval $T$. The Unified Law states that the distribution of waiting times between earthquakes depends only on this value. When the value of $x$ exceeds a well defined value, the earthquakes become dramatically less correlated.

Earthquakes can be thought of as a series of events being produced by a "process." This process generates a series of highly correlated earthquakes with a $T^{-\alpha}$ distribution. Visually, one might think of the process as the activity associated with

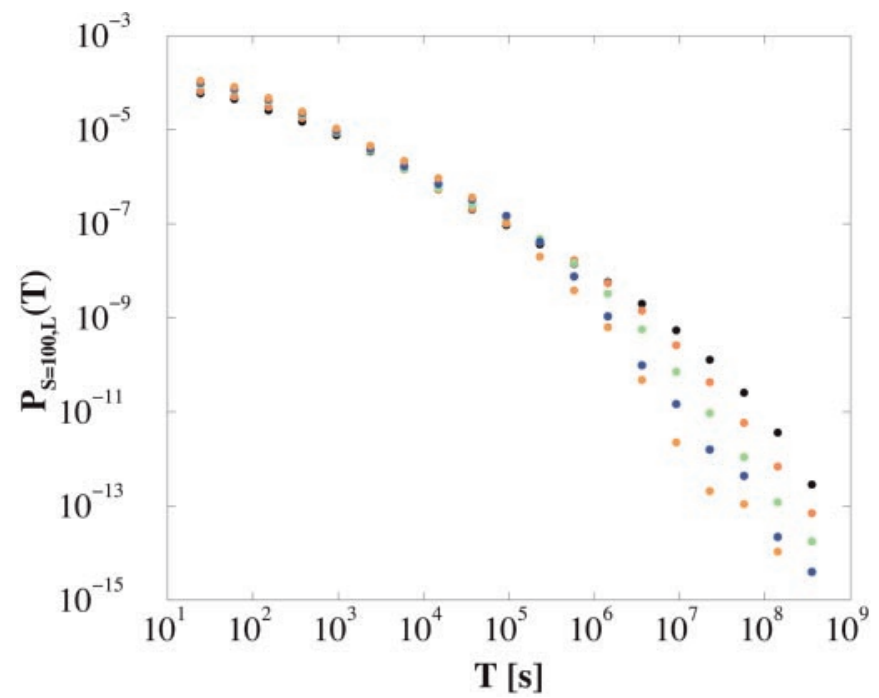

Fig. 5. First-return (interoccurrence) time distributions for $L=0.25^{\circ}, 0.5^{\circ}, 1^{\circ}$, $2^{\circ}$, and $4^{\circ}$ and $m=2$. The function follows a power law $P_{S=100, L}(T) \propto T^{-\alpha}$ where $\alpha \approx 1$. Note the Omori Law region decreases with increasing $L$.

dynamically changing fault-segment patterns. The Law indicates that the crust operates in the true SOC slow-driving regime (14), where the individual processes do not overlap. Because of the nonzero driving rate, several spatially separated processes are active simultaneously, and the kink in the curve represents the point where spatially distinct earthquakes, belonging to different processes, overlap. So, by choosing a small enough window in $T$ and $L$, one can ensure that only a single correlated process is sampled.

\section{Conclusion}

A number of key results have been achieved.

- Our data suggest that an earthquake is an SOC phenomenon, which has been anticipated for a long time. This

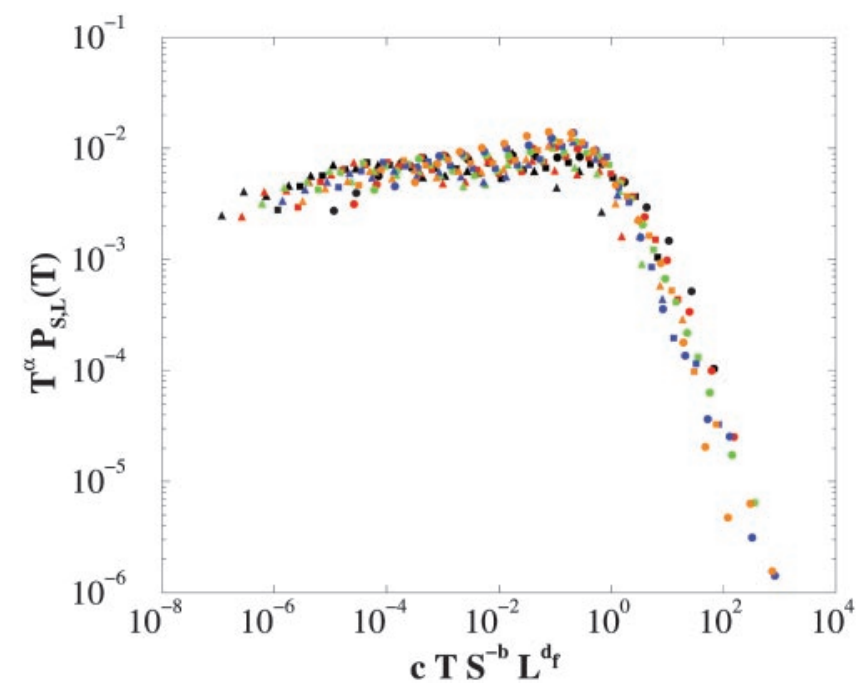

Fig. 6. The data in Fig. 3 with $T>38 \mathrm{~s}$ replotted with $T^{\alpha} P_{S, L}(T)$ as a function of the variable $x=c T S^{-b} L^{d f}, c=10^{-4}$. The Omori Law exponent $\alpha \approx 1$, Gutenberg-Richter value $b \approx 1$, and fractal dimension $d_{f} \approx 1.2$ have been used to collapse all of the data onto a single, unique curve $f(x)$. The curve is constant for $x<1$, corresponding to the correlated, Omori Law regime but decays fast for $x>1$, associated with uncorrelated events. 
strongly indicates that the current terminology of earthquakes is flawed, that there is essentially no difference between aftershocks and main shocks, and that the mechanism responsible for small events also is responsible for large events.

- We have proposed and verified a Unified Law for Earthquakes that describes the probability of interoccurrence times between earthquakes for a cutoff magnitude and region size. This Law links together the key areas of earthquake research, the Gutenberg-Richter Law, the Omori Law of aftershocks, and the fractal dimension of the fault, and if provides a new view of the nature of earthquakes.

- This Law has several consequences. Essentially, it expresses an earthquake as a sequence of hierarchical correlated processes, which, because of the nonzero driving force, overlaps with other spatially separated correlated processes for a large

1. Bak, P., Tang, C. \& Wiesenfeld, K. (1987) Phys. Rev. Lett. 59, 381-382.

2. Gutenberg, B. \& Richter, C. F. (1944) Bull. Seismol. Soc. Amer. 34, $185-188$.

3. Omori, F. (1895) J. College Sci. Imper. Univ. Tokyo 7, 111-200.

4. Okubo, P. G. \& Aki, K. (1987) J. Geophys. Res. 92, 345-355.

5. Gardner, J. K. \& Knopoff, L. (1974) Bull. Seismol. Soc. Am. 64, 1363-1367.

6. Ito, K. (1995) Phys. Rev. E Stat. Phys. Plasmas Fluids Relat. 52, 3232-3233.

7. Bak, P. (1997) How Nature Works (Oxford Univ. Press, Oxford). enough region or time. This view suggests that the Omori Law for aftershocks is a short-term time and small spatial limit of a general hierarchical phenomenon; it states that the only difference between a main shock and an aftershock is the size of the sampling window that one chooses. Fundamentally, a main shock and an aftershock are consequences of the same process.

We hope this work will provide a rigorous framework for viewing earthquake sequence distributions and give an alternative view of the nature of seismic events.

We thank C. Scholz for advising us on earthquake catalogs. P.B. acknowledges helpful discussions with M. Paczuski. K.C. gratefully acknowledges the financial support of the U.K. Engineering and Physical Sciences Research Council through Grants GR/R44683/01 and GR/ L95267/01.

8. Jensen, H. J. (1998) Self-Organized Criticality (Cambridge Univ. Press, Cambridge, U.K.)

9. Sornette, A. \& Sornette, D. (1989) Europhys. Lett. 9, 197-202.

10. Bak, P. \& Tang, C. (1989) J. Geophys. Res. 94, 15635-15637.

11. Ito, K. \& Matsuzaki, M. (1990) J. Geophys. Res. 95, 6853-6860.

12. The Southern California Seismographic Network Catalogs (1984) http:// www.scecdc.scec.org/ftp/catalogs/SCSN/.

13. Yeomans, J. M. (1992) Statistical Mechanics of Phase Transitions (Clarendon, Oxford).

14. Corral, A. \& Paczuski, M. (1999) Phys. Rev. Lett. 83, 572-575. 